\title{
Technetium-99m tetrofosmin: Use for myocardial perfusion imaging in the detection of coronary artery disease
}

\section{Helen Erica D’Arceuil}

Department of Radiology, Stanford University School of Medicine, California, USA
Correspondence: HE D'Arceuil Department of Radiology, HOI00, Nuclear Medicine Imaging Laboratory, Stanford University School of Medicine, 300 Pasteur Drive, Stanford, CA 94303, USA

$\mathrm{Tel}+$ I 650725 II 47

Email helen@nmr.mgh.harvard.edu
This article was published in the following Dove Press journal:

Reports in Medical Imaging

II August 2010

Number of times this article has been viewed
Abstract: Technetium-99m tetrofosmin is a radioactive tracer which is designed for myocardial perfusion imaging. Differential tracer uptake allows discrimination between normal and ischemic myocardium. Technetium-99m tetrofosmin has shown excellent utility as a safe, cost-effective, and technically sound agent for use in the diagnosis of coronary artery disease. This paper provides an overview of the available literature on clinical applications of technetium-99m tetrofosmin, and shows how this tracer has gained widespread acceptance as a diagnostic aid since its inception approximately 17 years ago. The various cardiac imaging modalities and imaging protocols which have employed this agent are also briefly reviewed.

Keywords: technetium-99m tetrofosmin, myocardial, viability, imaging

\section{Introduction}

Coronary artery disease (CAD), or coronary heart disease, manifests when there is narrowing of the small vessels or coronary arteries which supply blood to the heart. Possible complications of CAD are heart attack (myocardial infarction), heart failure (insufficient cardiac output), ischemic cardiomyopathy, unstable or stable angina, cardiac arrest, or sudden death. There are several well established nuclear imaging techniques for interrogation of cardiac structure, perfusion, and metabolism, for example, single photon computed tomography (SPECT), which may use a radiopharmaceutical such as technetium-99m tetrofosmin, technetium-99m sestamibi (MIBI), technetium-99m furifosmin, or thallium-201, and position emission tomography (PET) which generally uses a metabolic tracer, fluorodeoxyglucose $\left({ }^{18} \mathrm{FDG}\right)$. In addition, there are technical improvements in these imaging techniques, such as attenuation-corrected SPECT, dual isotope simultaneous acquisition (DISA) SPECT and echocardiography-gated SPECT, which have improved our ability to assess myocardial perfusion and/or metabolism accurately. Finally, in recent years, technical advances in magnetic resonance imaging (MRI) have made this modality a powerful tool for the diagnosis and characterization of heart disease and, in conjunction with technetium-99m tetrofosmin, myocardial perfusion imaging (MPI) has been used to identify myocardial tissue at risk. However, this review focuses on the use of technetium-99m tetrofosmin for diagnostic and prognostic myocardial scintigraphy, which provides a cost-effective tool for early detection of obstructive $\mathrm{CAD}$, in turn guiding clinical decision-making, and will also review data regarding other techniques which have contributed to unraveling the complexities of altered myocardial function. submit your manuscript | www.dovepress.com

Dovepress

7565 


\section{Development}

In 1993, Kelley et al reported on the synthesis of a new radiopharmaceutical, technetium-99m tetrofosmin, for use in MPI. ${ }^{1}$ The preclinical testing of this radiopharmaceutical showed that there was excellent uptake and retention in viable myocardium with efficient clearance from nontarget tissues, in particular the blood and liver. Technetium-99m tetrofosmin was launched in Japan in April 1994, in Europe in May 1994, and in the US in April 1996. Technetium-99m tetrofosmin is currently available under the brand name Myoview $^{\circledR}$ (GE Healthcare). This compound has gained widespread acceptance as one of the favored radionuclides for SPECT scintigraphy because it has favorable physical properties, is easy to manufacture, incurs low production costs, and is available as an easily reconstituted kit which allows simple and rapid preparation for patient dosing.

Prior to the release of technetium-99m tetrofosmin in Japan, there were some early published studies reporting the utility of this radiopharmaceutical for MPI in humans..$^{2-7}$ The first biodistribution, safety, and dosimetric study, during exercise and at rest, revealed no significant clinical concerns in normal volunteers. ${ }^{8}$ High quality diagnostic myocardial images from as early as five minutes up to several hours post-injection were obtained.

\section{Characteristics and pharmacokinetics}

Technetium-99m tetrofosmin is a cationic complex $\left[{ }^{99} \mathrm{Tc}\right.$ Tetrofosmin $\left.\mathrm{O}_{2}\right]^{+}$in which tetrofosmin is the functionalized (1,2-bis[bis(2-ethoxyethyl) phosphino] ethane) ligand. The chemical structure is shown in Figure 1. Technetium-99m

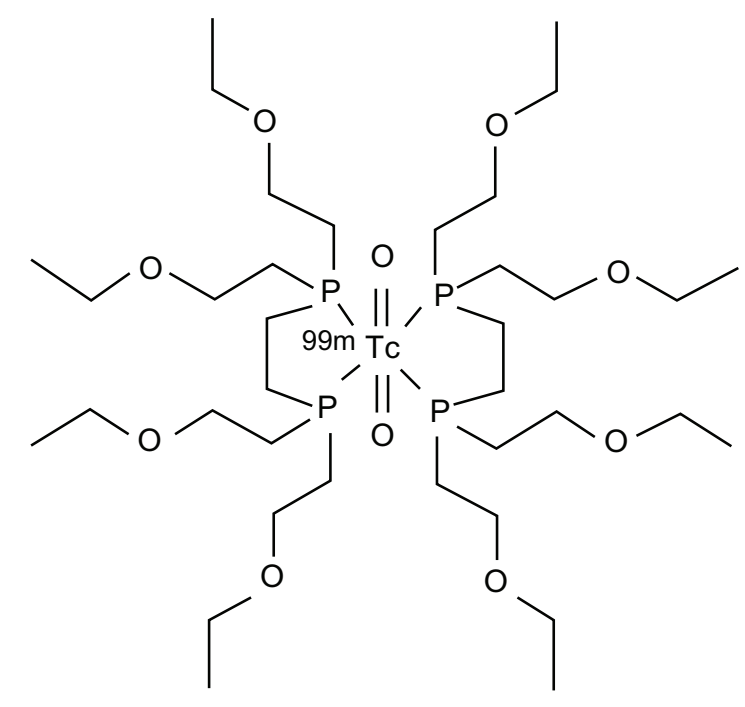

Figure I Chemical structure of ${ }^{99} \mathrm{Tc}$ Tetrofosmin. tetrofosmin, when injected intravenously, rapidly accumulates in the myocardium as well as nontarget organs (liver, kidney, spleen, and skeletal muscle). ${ }^{1,3}$ The first-pass extraction fraction in myocardium is 0.45 , and there is minimal clearance at three hours, with a typical myocardial uptake of up to $1.2 \%$ of the injected dose. ${ }^{8}$ There is faster clearance of the tracer from the myocardial wall during stress compared with at rest, it is efficiently cleared from the blood, and washout in nontarget organs is rapid at peak exercise. ${ }^{3}$ In addition, there is significant uptake of technetium-99m tetrofosmin in skeletal muscle and enhanced sequestration of the agent during exercise. ${ }^{8}$ Less than half of the administered dose of contrast agent is eliminated via the kidneys (approximately 40\%) and intestines (approximately $34 \%$ and $25 \%$ at rest and during exercise, respectively) within 48 hours after administration either during exercise or at rest.

\section{Myocardial perfusion imaging Mechanisms of myocardial uptake}

In human subjects, MPI is based on the assumption of a linear correlation between myocardial blood flow and tracer uptake. However, there is some controversy regarding the mechanism of uptake of technetium-99m tetrofosmin by the myocardial cells (cardiomyocytes). It has been suggested that technetium- $99 \mathrm{~m}$ tetrofosmin passes into the myocardium by a process of passive diffusion but, in a preclinical study in rats, Platts et al reported that tracer uptake was a metabolismdependent process which is not facilitated by cation channel transport. ${ }^{9}$ In contrast, in another preclinical study in guinea pigs, it was shown that technetium- $99 \mathrm{~m}$ tetrofosmin uptake is directly related to energy-dependent transport processes, as well as cellular ion homeostasis and mitochondrial membrane potentials. ${ }^{10}$ Hence cellular alterations that affect these processes will also allow technetium-99m tetrofosmin uptake in excess of flow-dependent accumulation in the myocardium.

It is generally accepted that once in the myocardium, there is little or no redistribution of technetium-99m tetrofosmin for at least 3-4 hours, and it washes out slowly as the patient assumes normal activity. ${ }^{8,11}$ Contrary to this, it has also been reported that the agent undergoes reverse redistribution (RR) within the myocardium over approximately three hours in patients with acute myocardial infarction, ${ }^{12,13}$ left bundle branch block, ${ }^{14}$ and hypertrophic cardiomyopathy. ${ }^{15}$ Reverse redistribution of the technetium-99m tetrofosmin tracer within the myocardium, after a single injection, was also observed in patients with acute myocardial infarction ${ }^{12}$ and was suggested to reflect myocardial viability because the myocardial segments that underwent RR showed improved 
wall motion one month later. ${ }^{12}$ Indeed, the same team of researchers suggested that RR may be due to retention of the tracer by dysfunctional cardiomyocytes in patients with hypertrophic cardiomyopathy. ${ }^{15}$ However, the finding of RR within the myocardium was made purely by visual inspection, and Hirata et al then suggested that, moving forward, it was necessary to perform systematic calculation of the regional tracer washout kinetics, ${ }^{13}$ an issue which continues to receive some attention.

\section{Timing of the scan}

After technetium-99m tetrofosmin has been injected, there is an optimal time window within which SPECT scintigraphy should be performed. Also, this timing will depend on whether the examination is performed at rest or during stress. Sridhara et $\mathrm{al}^{7}$ suggested that the optimal time for imaging was approximately 30 minutes post-injection while the patient was at rest and as early as five minutes after exercise. In addition, it was noted that, due to slow myocardial washout, quality diagnostic images can be acquired for up to four hours after injection. Early imaging, 10-15 minutes post injection Tc-99m-tetrofosmin, is certainly obtainable in a good majority of patients. However, in order to avoid image artifacts in the inferior myocardial wall (due to high signal intensity within the liver, myocardium/liver ratio of $0.4-0.5$ at 15 minutes post injection) Matsunari et al suggested that it may be best to image 1 hour later in patients with CAD. ${ }^{25}$

Nakajima et al used technetium-99m tetrofosmin MPI with a one-day protocol (rest and exercise) and showed that this agent had a good kinetic profile and, more importantly, good quality MPI images were obtained as early as 10 minutes post-contrast injection. ${ }^{6}$ However, the authors also suggested that imaging should be performed 45 minutes after injection to avoid image artifacts due to radionuclide accumulation within the liver.

The feasibility of imaging in the presence of high hepatic activity was also investigated by Matsunari et al and two radial image reconstruction formats were investigated. ${ }^{16}$ This study suggested early imaging at 10 minutes post-injection, but still reported some problems with image artifacts caused by high tracer activity within the liver which obscures part of the inferior myocardial wall, especially with the $180^{\circ}$ image reconstruction protocol. The authors recommended late imaging (one hour post-injection) in patients with CAD. ${ }^{16}$ This finding of inferior wall image artifacts is inconsistent with the findings of several authors, ${ }^{1,17,18}$ as well as those of a large Phase III clinical trial which claimed excellent heart:liver ratios at 15 minutes post-injection in both stress and rest regimens. ${ }^{19}$ Post-injection imaging at 15 minutes is currently advised by the manufacturers of technetium- $99 \mathrm{~m}$ tetrofosmin, and hence this is what is typically done clinically. However, it has been shown that when these artifacts occur, they can be reliably corrected by data post-processing. ${ }^{20}$

One has to bear in mind that technetium-99m tetrofosmin is given as a bolus injection and it was hypothesized that perhaps a change in the method of administration may improve its performance in identifying viable myocardium. The efficacy of an infusion versus a bolus injection protocol was demonstrated in a case study of a patient with a stenosed right coronary artery which resulted in a defect in the inferior myocardial wall. ${ }^{21}$ Imaging with a bolus of technetium-99m tetrofosmin (at rest) reported a severe wall defect, suggesting a myocardial infarction, which disappeared when an infusion of technetium-99m tetrofosmin was administered over the course of one hour one day later. The results from the pretreatment infusion examination were in agreement with those of the thallium-201 study at rest, and also with that of the post-treatment (revascularization) technetium-99m tetrofosmin examination. ${ }^{21}$ However, the authors suggested that infusion of tetrofosmin may be a more reliable administration protocol than bolus injection. This study has not had an influence on the current method for tracer administration which is via intravenous bolus injection.

\section{Initial clinical applications}

Identification of regional myocardial perfusion deficits and their severity is crucial for determining tissue at risk. In addition, being able to detect any change in these hypoperfused regions after intervention determines treatment efficacy and patients' long-term prognosis. The efficacy of technetium-99m tetrofosmin scintigraphy to evaluate myocardial tissue at risk in the acute period was demonstrated in patients with unstable angina and myocardial infarction. ${ }^{5}$ Patients were imaged in the acute period (day 3), then 30 days later, and it was found that acutely hypoperfused myocardial segments that had reperfused showed improved wall motion one month later compared with segments that had not recovered. In addition, there were several other clinical studies which demonstrated the utility of tetrofosmin for diagnosis of ischemic heart disease..$^{5-7,11,17,22-25}$

The most compelling body of evidence which supports the suitability and use of this new agent, technetium-99m tetrofosmin, for MPI was presented in a large multicenter Phase III clinical trial involving 250 patients from 10 centers both in the US and in Europe. ${ }^{19}$ The sensitivity and specificity of technetium-99m tetrofosmin for MPI was compared with that of thallium and coronary angiography. Judging by the imaging 
results, it was concluded that technetium-99m tetrofosmin images were of superior technical quality and relatively easier to interpret than thallium, although the sensitivity for detecting CAD was comparable between the two agents. It was also reported that the specificity for detecting CAD was low for both agents in this select population (patients referred for catherization either because of a previously abnormal noninvasive study or microvascular ischemia) but the normalcy rates were excellent. In fact, in a population such as this, the normalcy rate is the highly recommended index.

In light of these and other studies, it was not surprising that technetium-99m tetrofosmin was approved by the US Food and Drug Association (FDA) in April 1996 for use as a SPECT scintigraphy radionuclide tracer for the assessment of global and regional myocardial perfusion. In 2001, this radiopharmaceutical was approved by the FDA for use, in conjunction with a pharmacologic stress agent, for stress testing of patients who were unable to exercise. It was approved in the US just over two years later for the assessment of left ventricular function.

\section{Comparison with thallium and sestamibi}

Thallium-201 SPECT imaging at rest has been the most frequently utilized radionuclide technique for identifying viable myocardium and, as such, one has to evaluate how technetium-99m tetrofosmin measures up not only against thallium-201 but also with the other favored technetium tracer, MIBI. When injected intravenously, technetium-99m tetrofosmin and thallium-201 behave differently. Thallium-201 redistributes within the myocardium while technetium- $99 \mathrm{~m}$ tetrofosmin does not, hence two injections of thallium-201 are required for estimating myocardial viability based on the redistribution and the fill-in findings in the redistribution images. In a study by Kobayashi et al both technetium-99m tetrofosmin and thallium-201 scintigraphy performed comparably for detecting viable myocardium in a cohort of patients with chronic CAD. ${ }^{11}$ In another study of patients with CAD and left ventricular dysfunction, technetium-99m tetrofosmin MPI was shown to have underestimated myocardial viability compared with thallium-201, based on both visual comparison and quantitative assessment of the images. ${ }^{25}$ However, it is worth mentioning that the authors noted that tetrofosmin's stress distribution was higher than that of thallium-201, and hence there was less contrast between the myocardial defect and the normal wall. They cited the fact that technetium$99 \mathrm{~m}$ tetrofosmin is known to underestimate myocardial blood flow in a high-flow regimen, as demonstrated in a preclinical study by Sinusas et al. ${ }^{24}$ In addition, based on their quantitative estimation of the data, they hypothesized that defect identification can be enhanced for both tetrofosmin and thallium-201 if there is robust examination of the severity of the reduction in tracer activity within the regions of nonreversible defects. ${ }^{25}$ However, in a more recent clinical study, Matsunari et al also promote the advantages of technetium-99m tetrofosmin for detecting CAD and viable myocardial tissue, and reported that tetrofosmin scintigraphy had comparable diagnostic accuracy to that of thallium but with the added advantage of higher emission energy (140 $\mathrm{KeV}$ compared with 70-80 KeV for thallium) for reducing tissue photon attenuation. ${ }^{26}$

Like thallium-201, MIBI also exhibits lower myocardial tracer extraction than tetrofosmin, and also undergoes little redistribution within the myocardium. MIBI is another clinically approved and favored MPI radionuclide ${ }^{27}$ and, like tetrofosmin, allows simultaneous assessment of myocardial perfusion and left ventricular function. ${ }^{28}$ One might conclude that MIBI and tetrofosmin perform equally for MPI, however, in a study which compared the myocardial uptake of these agents, approximately one hour after stress, it was found that MIBI exhibited higher myocardial counts in both male and female patients. ${ }^{29}$ Similar results were also found in a preclinical study in pigs suggesting that MIBI may be a better flow tracer ${ }^{30}$ Later on, it was shown in a large clinical trial that there was no difference in the performance of MIBI, tetrofosmin, or thallium-201. ${ }^{31}$

It has been reported that the extraction and washout kinetics of MIBI, tetrofosmin, and thallium-201 vary depending on the ischemic state of the myocardium. Data from a recent preclinical study performed in isolated, perfused rat heart suggested that quantitative analysis of the tracers' kinetics may be useful for the evaluation of severe ischemia. ${ }^{32}$ In a similar study, using isolated rat hearts, it was reported that tetrofosmin showed the best correlation with infarct size, and concluded that this agent's kinetics may allow the best differentiation between viable and nonviable myocardium, while still retaining adequate activity for scintigraphy. ${ }^{33}$

\section{Flow sensitivity with technetium-99m tetrofosmin}

One might question whether technetium-99m tetrofosmin is an effective diagnostic agent over the pathophysiologic range of flows that exists during myocardial ischemia and/ or infarction. Experimental studies in dogs have sought to answer this important issue. ${ }^{24}$ In this model, the animal's proximal left anterior descending artery was completely 
occluded and subsequently a pharmacologic stress agent was administered, followed by technetium-99m tetrofosmin MPI. Radiolabeled microspheres were also administered for measurement of regional myocardial blood flow. Technetium$99 \mathrm{~m}$ tetrofosmin was found to be an accurate measure of flow over the range of flows produced during acute ischemia coupled with pharmacologic stress. However, in the high-flow regimen (flow rates greater than $2 \mathrm{~mL} / \mathrm{min}$ ) the flow rate was underestimated (similar to what has been seen with thallium) possibly due to reduced myocardial accumulation, which is independent of flow. The converse was found to be true in the low-flow regimen. Technetium-99m tetrofosmin's activity increased linearly with flow rates of up to $2 \mathrm{~mL} / \mathrm{min} / \mathrm{g}$. Discrimination of any differences in flow between ischemic and nonischemic myocardium was not possible during the first 15 minutes post-injection due to the confound of high liver activity. ${ }^{24}$

\section{Viability and stress testing}

Unstable angina is a CAD condition in which the heart is underperfused and where the stage is set for a future heart attack. If perfusion can be restored, a catastrophic cardiac event can be avoided. Therefore, myocardial viability testing is crucial for cardiac evaluation prior to and after reperfusion. Myocardial viability testing was initiated in a SPECT study, using technetium-99m tetrofosmin, in patients with unstable angina and acute myocardial infarction, and this study showed that segments with improvement in perfusion were accompanied by significant wall motion recovery compared with normal and unimproved myocardial segments. ${ }^{5}$ This early study supported the use of technetium-99m tetrofosmin SPECT scintigraphy for assessment of myocardial viability.

It is important to be able to perform both global and regional diagnostic evaluation of the heart to decide on the best therapy and management for individual patients. It is essential to determine whether there is sufficient viable myocardium following ischemic cardiomyopathy because, if there is a significant amount of scar tissue, revascularization is not advised. MPI when coupled with stress testing allows better identification of myocardial defects. Stress testing, whether by performing physical exercise or by pharmacologic induction (for patients unable to exercise) increases blood flow to the coronary arteries. Testing is then employed to determine how the heart responds to exertion. This may be a one- or two-day stress/rest protocol and requires two radiopharmaceutical injections, one at rest and another after stress.
It is also important to know the optimum time post-stress to perform MPI. Nakagawa et al performed both pharmacologic and exercise-induced stress testing in normal volunteers followed by technetium-99m tetrofosmin SPECT scintigraphy. ${ }^{34}$ Results from that study showed that the optimum time to perform MPI is immediately after stress (treadmill), but at least 40 minutes after a pharmacologic (dipyridamole) stress test. Another study, performed in a cohort of patients with known myocardial disease, compared the images acquired at one hour with those at four hours post-stress, as well those at one and four hours post-rest. ${ }^{35}$ The results from this study did not confirm the stable biokinetic profile which has been attributed to technetium-99m tetrofosmin. Instead, it was found that in this patient population there were significant differences in lesion severity, size, and number between one and four hours post-technetium-99m tetrofosmin SPECT. However, technetium-99m tetrofosmin scintigraphy showed quite different results in another study of patients with severe $\mathrm{CAD}^{36}$ where technetium-99m tetrofosmin was injected at peak exercise (dobutamine was given to patients who were unable to exercise), and technetium-99m tetrofosmin injected in the event of chest pain. Additionally, sublingual glyceryl trinitrate was administered followed by technetium-99m tetrofosmin injection. Improved detection of hypoperfused myocardial defects was reported after nitrate administration, but the authors cautioned against the widespread use of nitrate administration for MPI until there is further testing, especially in patients with less severe CAD.

Nitrates (as described above) and various other pharmacologic agents which increase regional coronary blood flow have been used in conjunction with scintigraphy to enable improved detection of ischemic, but viable, myocardial tissue based on the radiopharmaceutical accumulation profile. Nitrate administration followed by technetium-99m tetrofosmin SPECT imaging have been shown to improve identification of ischemic but still viable myocardium in patients with chronic ischemic LV dysfunction. ${ }^{37,38}$ Adenosine, another myocardial blood flow modulator, has also been used in conjunction with technetium99m tetrofosmin SPECT to discriminate ischemic from nonischemic myocardium in patients with mild to severe left ventricular systolic dysfunction (LVSD). ${ }^{39}$ While it was acknowledged that this technique was adequate for assessing patients with mild LVSD, it was suggested that a different imaging/stress approach may better suit patients with severe LVSD. A role has also been suggested for adesonine-technetium-99m tetrofosmin SPECT in the diagnosis of hypertrophic cardiomyopathy which predominantly affects the cardiac apex, a condition which may be missed by routine echocardiography. ${ }^{40}$ 


\section{Left ventricular dysfunction, stunned, and hibernating myocardium}

In the sequelae of chronic $\mathrm{CAD}$, left ventricular dysfunction is one of the most important determinants of prognosis. The gold standard for assessing myocardial viability is restoration of blood flow after left ventricular dysfunction and hence, improved function equals successful revascularization. It is important to identify patients with left ventricular dysfunction who have viable myocardium because they will be the best candidates for revascularization and also the patients most at risk for future cardiac ischemia. Dysfunctional myocardial vessels, if viable, have the ability to recover if blood flow is restored in a timely fashion, which affords the patient a better prognosis. There is some debate about whether technetium-99m tetrofosmin scintigraphy allows improved detection of stunned and/or hibernating myocardium. A study done by Adachi et $\mathrm{al}^{41}$ concluded that accurate detection of hibernating myocardium was difficult despite the reputed superior imaging capability of technetium-99m tetrofosmin SPECT. In addition, results from a preclinical study in pigs clearly showed that scarred myocardium, with no viability, accumulated a significant amount of technetium- $99 \mathrm{~m}$ tetrofosmin, as well as thallium-201, even in the absence of coronary collateral perfusion. ${ }^{42}$

The most recent data regarding myocardial viability imaging with tetrofosmin comes from a report of patients with acute myocardial infarction. This study compared (123)iodine-labeled 15-(p-iodophenyl)-3R,S-methyl pentadecanoic acid imaging ( ${ }^{123}$ I-BMIPP) combined with early and late technetium-99m tetrofosmin imaging or early and late thallium-201 imaging. ${ }^{43}$ This study concluded that the mismatch evident in the combined ${ }^{123}$ I-BMIPP and early technetium-99m tetrofosmin imaging predicted improvement in stunned myocardial wall motion, hence improved left ventricular function, better than any of the other imaging combinations. ${ }^{43}$ The problem now remains one of differentiating between viable myocardium and scarred myocardial tissue, in light of the controversial data presented so far.

\section{Technetium-99m tetrofosmin microspheres for blood flow measurement}

The use of radiolabeled microspheres for the measurement of blood flow is a well established technique, and the use of technetium-99m tetrofosmin microspheres as a blood flow tracer would afford a quantitative measure of the regional myocardial blood flow profile that directly relates to myocardial viability determined by MPI. A preclinical study in dogs was conducted to determine whether technetium-99m tetrofosmin microspheres could accurately depict regional differences in myocardial blood flow during sustained coronary artery occlusion. ${ }^{44}$ Data from this study showed that tracer activity was poorly correlated with reperfusion flow in nonviable artery segments (in spite of normal or hyperemic flow at the time of injection) but was strongly correlated with occlusion flow, even though the tracer was injected after reperfusion. This result clearly shows that technetium-99m tetrofosmin uptake is dependent on myocardial blood flow as well as cardiomyocyte viability (which reflects infarct size). ${ }^{44}$ These data also further validate the utility of technetium- $99 \mathrm{~m}$ tetrofosmin for assessing myocardial viability, in addition to assessing flow heterogeneity and infarct size during persistent coronary artery occlusion.

\section{Complementing perfusion information}

Our discussion will now turn to techniques used in conjunction with myocardial perfusion imaging using tetrofosmin, which have enhanced our ability to detect viable myocardial tissue and which have allowed differentiation between image artifacts, and normal and ischemic myocardial states.

\section{Cardiac gated SPECT}

The use of echocardiography-gated myocardial single photon emission tomography (gated SPET or gated SPECT) is reputed to allow discrimination of true perfusion defects from image artifacts and also gives information on left ventricular function. Echocardiography gating increases the accuracy of MPI and, along with attenuation and scatter compensation, enables more reliable artifact detection. In addition to calculating regional wall thickening and motion, indicators of systolic function can also be determined, ie, end diastolic and end systolic volumes and left ventricular ejection fraction. These systolic indicators are robust predictors of long-term prognosis. Forty percent of myocardial infarctions are accompanied by LVSD with or without heart failure. In addition, some patients may have asymptomatic LVSD due to injury of the cardiomyocytes, and this condition carries a high morbidity similar to that of patients with clinical heart failure.

Echocardiography-gated SPECT allows simultaneous evaluation of myocardial perfusion and, moreso, left ventricular perfusion and function within a single myocardial perfusion imaging examination. ${ }^{45,46}$ The added benefit of gating during the standard SPECT MPI protocol lies in the systolic functional analysis which can be done in order to reassess 
the infarcts which have been identified by standard perfusion imaging. This allows discrimination between true infarcts, scars, viable myocardium, and image artifacts. Technetium MPI tracers, such as technetium-99m tetrofosmin, are the preferred tracers for gated SPECT because larger doses can be administered as a result of the relatively short half-life (six hours compared with thallium which has a half-life of 73 hours), ultimately resulting in better image quality. ${ }^{2}$ While there are many gated SPECT studies which have reported the use of MIBI, there is also a wealth of data supporting the use of technetium-99m tetrofosmin. Gated SPECT during rest and exercise using technetium-99m tetrofosmin has been used for the assessment of myocardial viability and/ or left ventricular dysfunction. ${ }^{45,47-49}$ Gated SPECT has also been used in combination with other imaging modalities to enhance our ability to assess myocardial viability. Technetium$99 \mathrm{~m}$ tetrofosmin gated SPECT and ${ }^{18}$ FDG PET were used to differentiate successfully between viable and scarred myocardium. ${ }^{45-48}$ Gated SPECT has also been used in combination with BMIPP SPECT to identify myocardial perfusion/ metabolism mismatch ${ }^{50,51}$ and left ventricular function, ${ }^{52}$ thus enhancing the diagnostic accuracy of determining myocardial viability.

The assessment of regional wall motion can also indicate myocardial viability, ${ }^{53,54}$ but in a combined gated SPECTtechnetium-99m tetrofosmin study by Wahba et al the authors caution against the sole use of wall motion analysis as a viability indicator because post-ischemic stunning and hibernation may result in underestimation of myocardial viability. ${ }^{53}$ A quantitative measure of myocardial wall perfusion, thickening, and motion which can be determined automatically provides a much more objective method to assess wall function and viability. Technetium-99m tetrofosmin quantitative gated SPECT affords such a measure. ${ }^{55,56}$ The myocardium can be divided into segments and the average quantitative values of regional perfusion (percentage uptake, \%), wall motion (mm), and wall thickening (\%) determined using software which automatically segments, quantifies, and analyses static, gated short-axis myocardial perfusion SPECT images. A wall motion score can then be defined which represents a range of motions, normal through to severe asynergy, and affords a quantitative measure of viability.

\section{Dual tracer measurements}

Chronically hypoperfused myocardium which exhibits abnormalities in its ability to contract is classified as hibernating myocardium. However, hibernation may be displayed by acutely ischemic myocardium as well. Being able to distinguish between hibernating, stunned, and scarred myocardium resulting from left ventricular dysfunction is important for patient management and also to determine outcome. DISA is a technique which allows metabolic imaging $\left({ }^{18}\right.$ FDG SPECT) as well as regional perfusion assessment (technetium-99m tetrofosmin) and saves examination time compared with sequential perfusion/FDG imaging. In addition, this technique generates images which are perfectly aligned so that metabolism/perfusion match or mismatch, and hence myocardial viability, are easily appreciated. Viable myocardium is defined by both normal metabolism and perfusion, while severely decreased perfusion coupled with increased metabolism describes the mismatch condition. The mismatch of FDG metabolism and blood flow is indicative of hibernating myocardium which will recover function after revascularization. The feasibility of using DISA technology for evaluating myocardial viability has been demonstrated..$^{45,57-59}$ In addition, results from a study which utilized acipimox, which lowers free fatty acid levels and hence increases glucose utilization by the myocardium, showed that all images were of diagnostic quality, even though the images generated by DISA were less sharp compared with the technetium-99m tetrofosmin SPECT images. ${ }^{60}$ The authors concluded that the technetium99m tetrofosmin DISA SPECT protocol was well suited for routine clinical assessment of myocardial viability.

\section{Dobutamine and contractile reserve}

The primary mode of action of dobutamine (a sympathomimetic drug used in the treatment of heart failure and cardiogenic shock) is stimulation of the $\beta_{1}$-adrenoceptors of the heart, thereby increasing contractility and cardiac output. Low-dose dobutamine echocardiography (LDDE) serves to test the heart's contractile reserve which is thought to be a predictive indicator of cardiac resynchronization therapy outcome. In a study, which was designed to evaluate normal left ventricular function, normal volunteers were given low-dose dobutamine in addition to beta-blockers, and significant increases in regional and global left ventricular function were measured. ${ }^{54}$ In a study by Everaert et al myocardial defect severity was evaluated using technetium-99m tetrofosmin gated SPECT in combination with a metabolic tracer, BMIPP, at rest and post-dobutamine infusion. ${ }^{51}$ Regions of myocardial metabolism-perfusion match-mismatch, reflecting regions of viable/ nonviable myocardium, were identified. Functional recovery of left ventricular hibernating myocardium post-revascularization was also investigated using technetium-99m tetrofosmin SPECT or LDDE in combination with trimetazidine (TMZ, which, like acipimox, improves myocardial glucose utilization via inhibition of fatty acid metabolism).${ }^{61}$ The technetium-99m 
tetrofosmin SPECT-TMZ combination reported improved diagnostic accuracy compared with LDDE. However, a recent study which compared LDDE with DISA technetium-99m tetrofosmin and ${ }^{18}$ FDG SPECT showed that DISA was superior to LDDE in terms of its sensitivity in detecting viable myocardium, but LDEE had higher specificity. ${ }^{62}$ The authors suggest that a combination of the two techniques may improve the detection of myocardial viability in patients with acute myocardial infarction.

\section{Magnetic resonance imaging approaches}

Cardiac MRI is a technique which provides high resolution anatomic images of the heart. Contrast-enhanced cardiac MRI, which uses a gadolinium contrast agent, informs about perfusion within the myocardium within tens of seconds after the agent is administered. In regions of the myocardium where there is delayed accumulation (after approximately five minutes) of contrast agent, referred to as late enhancement, this accumulation is reputed to reflect irreversible damage and is highly sensitive for detecting and characterizing myocardial scar tissue and allowing distinction between subendocardial and transmural scars. However, this enhancement is not specific to ischemic damage and can also occur in regions of myocardial fibrosis, inflammation, and edema. As such, technetium-99m tetrofosmin SPECT, which is an established technique that provides myocardial viability information, can be combined and compared with contrast-enhanced cardiac MRI in order to validate data regarding myocardial area at risk and infarct volume. In a study by Carlsson et al that investigated patients who had first-time myocardial infarction with ST-segment elevation, there was no significant difference in the performance of the two techniques for identifying myocardial tissue at risk both before and for up to one week after reperfusion. ${ }^{63}$ In addition, the final infarct size, and hence the volume of salvaged myocardium, was easily quantified from the contrast-enhanced cardiac MRI.

One must bear in mind though that some viable myocardial tissue that shows no MRI contrast enhancement may still be mildly or moderately ischemic. This issue can be resolved by using a technique which gives both metabolic and perfusion information. Nuclear scintigraphy using technetium-99m tetrofosmin and ${ }^{18}$ FDG SPECT was compared with contrast-enhanced MRI to determine each technique's ability to determine myocardial viability. ${ }^{64}$ It was found that in a population of patients with severe left ventricular dysfunction, there was good agreement between the two techniques for accurately distinguishing myocardial segments with transmural scar tissue. However, in segments with subendocardial scar tissue, the nonenhanced epicardial rim seen on MRI contained either normal or ischemically compromised myocardium, showing that these two techniques provide different information about myocardial viability. This study shows that contrast-enhanced MRI does not inform on the pathophysiology of nonscarred myocardial tissue or its functional recovery after revascularization. In addition, this study also shows the superiority of the combined technetium$99 \mathrm{~m}$ tetrofosmin and ${ }^{18}$ FDG SPECT techniques which give information respectively about tissue perfusion and glucose consumption, which is critical for distinguishing between the different types of viable myocardial tissue, ie, stunned and hibernating myocardial tissue. However, there was a lack of follow-up data after reperfusion which would have informed about long-term tissue outcome. In terms of its utility in determining the long-term fate of salvaged myocardial tissue, there has been a recent report of a study of patients with ST-segment elevation acute myocardial infarction, which showed that greatly improved technetium-99m tetrofosmin myocardial uptake immediately after treatment heralded better recovery of left ventricular function and smaller final infarct size (one month after emergent percutaneous coronary intervention). ${ }^{65}$

\section{Summary}

The amount of salvaged myocardial tissue and the functional status of this tissue is clearly the most important factor determining patient outcome after an ischemic cardiac event. MPI using the technetium-99m tetrofosmin tracer, whether alone or in combination with blood flow-modulating drugs and/or other perfusion and metabolite imaging techniques, has no doubt played a major role in the evaluation of myocardial viability. In summary, MPI using technetium-99m tetrofosmin is among the favored radionuclides for MPI. In the clinic, technetium-99m tetrofosmin is commonly used as a robust diagnostic agent both with standard enhanced MPI modalities as well as in combination with modalities which measure myocardial metabolism. It has a proven record of utility for the assessment of left ventricular dysfunction and myocardial viability. However, its role as possibly the best agent for use in determining the final infarct extent is now emerging.

\section{Disclosure}

The author reports no conflict of interest in this work.

\section{References}

1. Kelly JD, Forster AM, Higley B, et al. Technetium-99m-tetrofosmin as a new radiopharmaceutical for myocardial perfusion imaging. $J \mathrm{Nucl}$ Med. 1993;34(2):222-227. 
2. Adachi I, Sugioka Y, Tanaka Y, et al. [Clinical efficacy of 99mTc-tetrofosmin myocardial scintigraphy - comparison to 201Tl myocardial scintigraphy]. Kaku Igaku. 1993;30(4):351-362. Japanese.

3. Inoue Y, Machida K, Honda N, et al. [Clearance of 99mTc-Tetrofosmin from the myocardium and the adjacent organs]. Kaku Igaku. 1993;30(3): 313-316.

4. Inoue Y, Machida K, Honda N, et al. [Comparison between 180 degrees and 360 degrees data collection in 99mTc-Tetrofosmin SPECT of the myocardium]. Kaku Igaku. 1993;30(1):85-88. Japanese.

5. Matsuo H, Watanabe S, Nishida Y, et al. Assessment of area at risk and efficacy of treatment in patients with acute coronary syndrome using 99mTc tetrofosmin imaging in humans. Ann Nucl Med. 1993;7(4): 231-238.

6. Nakajima K, Taki J, Shuke N, Bunko H, Takata S, Hisada K. Myocardial perfusion imaging and dynamic analysis with technetium-99m tetrofosmin. J Nucl Med. 1993;34(9):1478-1484.

7. Sridhara BS, Braat S, Rigo P, Itti R, Cload P, Lahiri A. Comparison of myocardial perfusion imaging with technetium-99m tetrofosmin versus thallium201 in coronary artery disease. Am J Cardiol. 1993;72(14):1015-1019.

8. Higley B, Smith FW, Smith T, et al. Technetium-99m-1,2-bis[bis(2ethoxyethyl) phosphino]ethane: human biodistribution, dosimetry and safety of a new myocardial perfusion imaging agent. $J$ Nucl Med 1993;34(1):30-38.

9. Platts EA, North TL, Pickett RD, Kelly JD. Mechanism of uptake of technetium-tetrofosmin. I: Uptake into isolated adult rat ventricular myocytes and subcellular localization. J Nucl Cardiol. 1995;2(4):317-326.

10. Schaefer WM, Nowak B, Kaiser HJ, et al. Comparison of microsphereequivalent blood flow (15O-water PET) and relative perfusion $(99 \mathrm{mTc}-$ tetrofosmin SPECT) in myocardium showing metabolism-perfusion mismatch. J Nucl Med. 2003;44(1):33-39.

11. Kobayashi H, Kawaguchi M, Oka T, et al. [Identification of viable myocardium using 99mTc-tetrofosmin scintigraphy - comparison with 201Tl redistribution-reinjection images]. Kaku Igaku. 1995;32(4):367-375. Japanese.

12. Sugihara H, Nakagawa T, Yamashita E, et al. Reverse redistribution of Tc-99m-tetrofosmin in patients with acute myocardial infarction. Ann Nucl Med. 1999;13(1):43-47.

13. Hirata $\mathrm{Y}$, Takamiya M, Kinoshita N, et al. Interpretation of reverse redistribution of $99 \mathrm{mTc}$-tetrofosmin in patients with acute myocardial infarction. Eur J Nucl Med Mol Imaging. 2002;29(12):1594-1599.

14. Sugihara H, Kinoshita N, Adachi Y, et al. Early and delayed Tc-99mtetrofosmin myocardial SPECT in patients with left bundle branch block. Ann Nucl Med. 1998;12(5):281-286.

15. Sugihara H, Taniguchi $Y$, Kinoshita N, et al. Reverse redistribution of Tc-99m-tetrofosmin in exercise myocardial SPECT in patients with hypertrophic cardiomyopathy. Ann Nucl Med. 1998;12(5):287-292.

16. Matsunari I, Tanishima Y, Taki J, et al. Early and delayed technetium99m-tetrofosmin myocardial SPECT compared in normal volunteers J Nucl Med. 1996;37(10):1622-1626.

17. Sridhara B, Sochor H, Rigo P, et al. Myocardial single-photon emission computed tomographic imaging with technetium $99 \mathrm{~m}$ tetrofosmin: stress-rest imaging with same-day and separate-day rest imaging. $J \mathrm{NuCl}$ Cardiol. 1994;1(2 Pt 1):138-143.

18. Takahashi N, Reinhardt CP, Marcel R, Leppo JA. Myocardial uptake of 99mTc-tetrofosmin, sestamibi, and 201Tl in a model of acute coronary reperfusion. Circulation. 1996;94(10):2605-2613.

19. Zaret BL, Rigo P, Wackers FJ, et al. Myocardial perfusion imaging with $99 \mathrm{mTc}$ tetrofosmin. Comparison to $201 \mathrm{Tl}$ imaging and coronary angiography in a phase III multicenter trial. Tetrofosmin International Trial Study Group. Circulation. 1995;91(2):313-319.

20. Nuyts J, Dupont P, Van den Maegdenbergh V, Vleugels S, Suetens P, Mortelmans L. A study of the liver-heart artifact in emission tomography. J Nucl Med. 1995;36(1):133-139.

21. Derebek E, Kozan O, Durak H, et al. Infusion versus bolus injection of Tc-99m tetrofosmin in the identification of viable myocardium. Clin Nucl Med. 1998;23(3):146-149.
22. Braat SH, Leclercq B, Itti R, Lahiri A, Sridhara B, Rigo P. Myocardial imaging with technetium-99m-tetrofosmin: comparison of one-day and two-day protocols. J Nucl Med. 1994;35(10):1581-1585.

23. Ohtsuki K, Sugihara H, Taniguchi Y, et al. [Evaluation of myocardial perfusion and ventricular shape in hypertrophic cardiomyopathy using 99mTc-tetrofosmin scintigraphy: comparison with 201Tl myocardial scintigraphy]. Kaku Igaku. 1994;31(4):309-318. Japanese.

24. Sinusas AJ, Shi Q, Saltzberg MT, et al. Technetium-99m-tetrofosmin to assess myocardial blood flow: experimental validation in an intact canine model of ischemia. $J$ Nucl Med. 1994;35(4): 664-671.

25. Matsunari I, Fujino S, Taki J, et al. Myocardial viability assessment with technetium-99m-tetrofosmin and thallium-201 reinjection in coronary artery disease. J Nucl Med. 1995;36(11):1961-1967.

26. Matsunari I, Fujino S, Taki J, et al. Comparison of defect size between thallium-201 and technetium-99m tetrofosmin myocardial single-photon emission computed tomography in patients with single-vessel coronary artery disease. Am J Cardiol. 1996;77(5):350-354.

27. Maes AF, Borgers M, Flameng W, et al. Assessment of myocardial viability in chronic coronary artery disease using technetium- $99 \mathrm{~m}$ sestamibi SPECT. Correlation with histologic and positron emission tomographic studies and functional follow-up. J Am Coll Cardiol. 1997; 29(1):62-68.

28. Villanueva-Meyer J, Mena I, Narahara KA. Simultaneous assessment of left ventricular wall motion and myocardial perfusion with technetium99m-methoxy isobutyl isonitrile at stress and rest in patients with angina: comparison with thallium-201 SPECT. J Nucl Med. 1990;31(4): $457-463$.

29. Gremillet E, Champailler A. Comparative myocardial uptake of technetium-99m sestamibi and technetium-99m tetrofosmin one hour after stress injection. Eur J Nucl Med. 1998;25(11):1502-1510.

30. Matsunari I, Haas F, Nguyen NT, et al. Comparison of sestamibi, tetrofosmin, and Q12 retention in porcine myocardium. J Nucl Med. 2001; 42(5):818-823.

31. Kapur A, Latus KA, Davies G, et al. A comparison of three radionuclide myocardial perfusion tracers in clinical practice: the ROBUST study. Eur J Nucl Med Mol Imaging. 2002;29(12): 1608-1616.

32. Fukushima K, Momose M, Kondo C, Kusakabe K, Kasanuki H. Myocardial kinetics of (201)Thallium, (99m)Tc-tetrofosmin, and (99m) Tc-sestamibi in an acute ischemia-reperfusion model using isolated rat heart. Ann Nucl Med. 2007;21(5):267-273.

33. Okada DR, Liu Z, Beju D, Okada RD, Johnson G, 3rd. Monocationic radiotracer kinetics and myocardial infarct size: a perfused rat heart study. Ann Nucl Med. 200;22(7):617-627.

34. Nakagawa S, Kimura M, Kurimura K, Igarashi A, Ooba Y. [Optimal protocol for $99 \mathrm{mTc}$-tetrofosmin myocardial SPECT imaging with exercise or dipyridamole stress test and the characteristics of bullseye normal file]. Kaku Igaku. 1996;33(10):1073-1082. Japanese.

35. Pillay M, Cox PH, Schonfeld DH, Mathews S. Tc-99m tetrofosmin myocardial distribution 4 hours post injection. Int $J$ Card Imaging. 1996;12(2):127-135.

36. Thorley PJ, Bloomer TN, Sheard KL, Sivananthan UM. The use of GTN to improve the detection of ischaemic myocardium using 99Tcmtetrofosmin. Nucl Med Commun. 1996;17(8):669-674.

37. He W, Acampa W, Mainolfi C, et al. Tc-99m tetrofosmin tomography after nitrate administration in patients with ischemic left ventricular dysfunction: relation to metabolic imaging by PET. J Nucl Cardiol. 2003; 10(6):599-606.

38. Flotats A, Carrio I, Estorch M, et al. Nitrate administration to enhance the detection of myocardial viability by technetium-99m tetrofosmin single-photon emission tomography. Eur J Nucl Med. 1997;24(7): 767-773.

39. Her SH, Yoon HJ, Lee JM, et al. Adenosine Tc-99m tetrofosmin SPECT in differentiation of ischemic from nonischemic cardiomyopathy in patients with LV systolic dysfunction. Clin Nucl Med. 2008;33(7): 459-463. 
40. Irwin RB, Arumugam P, Khattar RS. Incidental detection of apical hypertrophic cardiomyopathy by myocardial perfusion imaging. $\mathrm{Nucl}$ Med Commun. 2010;31(4):286-293.

41. Adachi I, Hou N, Komori T, et al. [Usefulness of 99mTc-tetrofosmin myocardial scintigraphy before and after coronary intervention]. Kaku Igaku. 1997;34(6):363-369. Japanese.

42. Cinca J, Garcia-Burillo A, Carreno A, Castell J, Warren M, CandellRiera J, et al. Differential uptake of myocardial perfusion radiotracers in normal, infarcted, and acutely ischemic peri-infarction myocardium.

43. Ueda T, Toyama T, Seki R, et al. Prediction of functional outcome in stunned myocardium after myocardial infarction using BMIPP and tetrofosmin imaging. J Int Med Res. 2009;37(2):367-377.

44. Glover DK, Ruiz M, Koplan BA, Watson DD, Beller GA. 99mTc-tetrofosmin assessment of myocardial perfusion and viability in canine models of coronary occlusion and reperfusion. J Nucl Med. 1999;40(1):142-149.

45. Kuwabara Y, Watanabe S, Nakaya J, et al. Functional evaluation of myocardial viability by $99 \mathrm{mTc}$ tetrofosmin gated SPECT - a quantitative comparison with $18 \mathrm{~F}$ fluorodeoxyglucose positron emission CT (18F FDG PET). Ann Nucl Med. 1999;13(3):135-140.

46. Bavelaar-Croon CD, Pauwels EK, van der Wall EE. Gated single-photon emission computed tomographic myocardial imaging: a new tool in clinical cardiology. Am Heart J. 2001;141(3):383-390.

47. Stollfuss JC, Haas F, Matsunari I, et al. 99mTc-tetrofosmin SPECT for prediction of functional recovery defined by MRI in patients with severe left ventricular dysfunction: additional value of gated SPECT. J Nucl Med. 1999;40(11):1824-1831.

48. Maruyama A, Hasegawa S, Paul AK, et al. Myocardial viability assessment with gated SPECT Tc-99m tetrofosmin \% wall thickening: comparison with F-18 FDG-PET. Ann Nucl Med. 2002;16(1):25-32.

49. Gunning MG, Anagnostopoulos C, Davies G, et al. Simultaneous assessment of myocardial viability and function for the detection of hibernating myocardium using ECG-gated 99Tcm-tetrofosmin emission tomography: a comparison with $201 \mathrm{Tl}$ emission tomography combined with cine magnetic resonance imaging. Nucl Med Commun. 1999; 20(3):209-214.

50. Zhao C, Shuke N, Okizaki A, et al. Comparison of myocardial fatty acid metabolism with left ventricular function and perfusion in cardiomyopathies: by 123I-BMIPP SPECT and 99mTc-tetrofosmin electrocardiographically gated SPECT. Ann Nucl Med. 2003;17(7): 541-548.

51. Everaert H, Vanhove C, Franken PR. Assessment of perfusion, function, dose dobutamine tetrofosmin gated SPECT perfusion scintigraphy and BMIPP SPECT imaging. J Nucl Cardiol. 2000;7(1):29-36.

52. Nanasato $M, A$ Ando $A$, Isobe $S$, et al. Evaluation of left ventricular function using electrocardiographically gated myocardial SPECT with (123) I-labeled fatty acid analog. J Nucl Med. 2001;42(12):1747-1756.

53. Wahba FF, Bavelaar-Croon CD, Baur LH, et al. Detection of residual wall motion after sustained myocardial infarction by gated $99 \mathrm{Tcm}-$ tetrofosmin SPECT: a comparison with echocardiography. Nucl Med Commun. 2001;22(2):175-182. Cardiovasc Res. 1998;38(1):91-977. and myocardial metabolism after infarction with a combination of low-

54. Everaert H, Vanhove C, Franken PR. Effect of beta-blockade on low-dose dobutamine-induced changes in left ventricular function in healthy volunteers: assessment by gated SPET myocardial perfusion scintigraphy. Eur J Nucl Med. 2000;27(4):419-424.

55. Mabuchi M, Kubo N, Morita K, et al. Prediction of functional recovery after coronary bypass surgery using quantitative gated myocardial perfusion SPECT. Nucl Med Commun. 2003;24(6):625-631.

56. Kasama S, Toyama T, Kumakura H, et al. Low-dose dobutamine stress $99 \mathrm{mTc}$ tetrofosmin quantitative gated SPECT performed during the acute phase of myocardial infarction predicts subsequent myocardial viability and detects stunned myocardium. Nucl Med Commun. 2003; 24(2):167-174.

57. Sozzi FB, Poldermans D, Bax JJ, et al. Improved identification of viable myocardium using second harmonic imaging during dobutamine stress echocardiography. Heart. 2001;86(6):672-678.

58. Rambaldi R, Poldermans D, Bax JJ, et al. Dobutamine stress echocardiography and technetium-99m-tetrofosmin/fluorine 18-fluorodeoxyglucose single-photon emission computed tomography and influence of resting ejection fraction to assess myocardial viability in patients with severe left ventricular dysfunction and healed myocardial infarction. Am J Cardiol. 1999;84(2):130-134.

59. Fukuchi K, Katafuchi T, Fukushima K, et al. Estimation of myocardial perfusion and viability using simultaneous $99 \mathrm{mTc}$-tetrofosmin - FDG collimated SPECT. J Nucl Med. 2000;41(8):1318-1323.

60. Kam BL, Valkema R, Poldermans D, et al. Feasibility and image quality of dual-isotope SPECT using 18F-FDG and (99m)Tctetrofosmin after acipimox administration. J Nucl Med. 2003;44(2): 140-145.

61. Feola M, Biggi A, Francini A, et al. Placebo or trimetazidine (99m)Tc tetrofosmin myocardial SPECT and low-dose dobutamine echocardiography in hibernating myocardium. Arch Med Res. 2006;37(1): 117-122.

62. Fang LG, Chen LB, Li F, Zhu WL, Fang Q. [Comparison of low-dose dobutamine echocardiography and dual-isotope emission simultaneous myocardial perfusion acquisition for myocardial viability assessment]. Zhonghua Xin Xue Guan Bing Za Zhi. 2007;35(4):325-328. Chinese.

63. Carlsson M, Ubachs JF, Hedstrom E, Heiberg E, Jovinge S, Arheden H. Myocardium at risk after acute infarction in humans on cardiac magnetic resonance: quantitative assessment during follow-up and validation with single-photon emission computed tomography. JACC Cardiovasc Imaging. 2009;2(5):569-576.

64. Roes SD, Kaandorp TA, Marsan NA, et al. Agreement and disagreement between contrast-enhanced magnetic resonance imaging and nuclear imaging for assessment of myocardial viability. Eur J Nucl Med Mol Imaging. 2009;36(4):594-601.

65. Yoshida S, Nakamura S, Sugiura T, et al. Factors associated with myocardial salvage immediately after emergent percutaneous coronary intervention in patients with ST-elevation acute myocardial infarction. Ann Nucl Med. 2009;23(4):383-390.

Reports in Medical Imaging

\section{Publish your work in this journal}

Reports in Medical Imaging is an international, peer-reviewed, open access journal publishing original research, reports, reviews and commentaries on all areas of medical imaging. The manuscript management system is completely online and includes a very quick and fair peer-review system, which is all easy to use.

Submit your manuscript here: http://www.dovepress.com/reports-in-medical-imaging-journal
Dovepress

Visit http://www.dovepress.com/testimonials.php to read real quotes from published authors. 